\title{
BEST CONSTANT FOR THE RATIO OF THE FIRST TWO EIGENVALUES OF ONE-DIMENSIONAL SCHRÖDINGER OPERATORS WITH POSITIVE POTENTIALS
}

\author{
MARK S. ASHBAUGH AND RAFAEL BENGURIA
}

\begin{abstract}
We prove the optimal upper bound $\lambda_{2} / \lambda_{1} \leq 4$ for the ratio of the first two eigenvalues of one-dimensional Schrödinger operators with nonnegative potentials. Equality holds if and only if the potential vanishes identically.
\end{abstract}

In this note we improve upon the one-dimensional portion of a theorem of Singer, Wong, Yau, and Yau [7] concerning the first two eigenvalues of a Schrödinger operator on a bounded domain with Dirichlet boundary conditions. Our result is best possible for the class of nonnegative' potentials; in addition, it is an easy consequence of a commutation formula and the Rayleigh-Ritz inequality.

THEOREM. Let $[a, b]$ be a finite interval and let $V$ be a nonnegative real-valued function in $L^{1}(a, b)$. Let $H=-d^{2} / d x^{2}+V(x)$ with Dirichlet boundary conditions act on $L^{2}(a, b)$, and let $\lambda_{1}[V]$ and $\lambda_{2}[V]$ denote the first two eigenvalues of $H$. Then $\lambda_{2}[V] / \lambda_{1}[V] \leq 4$ with equality if and only if $V=0$.

PROOF. Let $u_{1}$ be the groundstate eigenfunction of $H$, i.e. $u_{1}$ is in the domain of $H$ and $H u_{1}=\lambda_{1} u_{1}$. By the commutation formula (see, in particular, [3] and also $[\mathbf{1}, \mathbf{2}, \mathbf{5}])$, the operator $\tilde{H}=-d^{2} / d x^{2}+V-2\left(u_{1}^{\prime} / u_{1}\right)^{\prime}$ has the same spectrum as $H$ except for $\lambda_{1}$. Thus we can obtain an upper bound for $\lambda_{2}$ by using the Rayleigh-Ritz inequality on $\tilde{H}$. Taking $u_{1}^{2}$ as our trial function we find $\tilde{H} u_{1}^{2}=$ $-4 u_{1} u_{1}^{\prime \prime}+V u_{1}^{2}=4 \lambda_{1} u_{1}^{2}-3 V u_{1}^{2}$ and hence

$$
\lambda_{2} \leq 4 \lambda_{1}-3 \frac{\int_{a}^{b} V u_{1}^{4} d x}{\int_{a}^{b} u_{1}^{4} d x} .
$$

This shows that $\lambda_{2} / \lambda_{1} \leq 4$ for nonnegative potentials. Moreover, equality holds if and only if $V=0$ since $u_{1}$ is positive and continuous on $(a, b)$.

REMARKS. 1. The choice of $u_{1}^{2}$ as the trial function was motivated by the fact that $u_{1}^{2}$ is the groundstate wavefunction of $\tilde{H}$ when $V=0$.

2. $H$ above is to be interpreted in the sense of quadratic forms following $[\mathbf{3}]$, for example.

Received by the editors February 10, 1986.

1980 Mathematics Subject Classification. Primary 34B25.

Key words and phrases. Schrödinger operators, ratios of eigenvalues, commutation formula.

The first author was partially supported by grants from the Research Council of the Graduate School, University of Missouri-Columbia and the Programa de las Naciones Unidas para el Desarrollo (PNUD grant CHI-84-005).

The second author was partially supported by the Departamento de Investigación y Bibliotecas de la Universidad de Chile (Grant E-1959-8522). 
3. Our result reads $\lambda_{2}-\lambda_{1} \leq 3 \lambda_{1}$ when stated as a result about $\lambda_{2}-\lambda_{1}$. This improves upon the bound $\lambda_{2}-\lambda_{1} \leq 4 \lambda_{1}$ found in [7 and 1]. Such a bound (for general dimension) had also been obtained by Harrell [4] based on the work of Payne, Pólya, and Weinberger [6]. The paper [1] derived the bound $\lambda_{2}-\lambda_{1} \leq 4 \lambda_{1}$ using the commutation formula and the Rayleigh-Ritz inequality in much the way we do above but with $u_{1}$ rather than $u_{1}^{2}$ as trial function.

4. We note that formula (1) holds for all $V$, not just for nonnegative $V$. In addition, we would expect formula (1) and the inequality $\lambda_{2} / \lambda_{1} \leq 4$ to hold for singular potentials $\left(V \in L_{\text {loc }}^{1}(a, b)\right.$, say) and also for problems on the line and half-line where $V(x) \rightarrow \infty$ as $x \rightarrow \pm \infty$. Of course, we always assume that Dirichlet boundary conditions are imposed at limit circle endpoints. Formally, all these results follow by commutation but one needs to take care that the operator $\tilde{H}$ generated in this way is well defined, selfadjoint, and has Dirichlet boundary conditions (when needed).

ACKNOWLEDGMENTS. It is a pleasure to thank Evans Harrell for communicating his results on eigenvalue differences to us. One of us (M. A.) would like to thank the members of the Departamento de Física of the Universidad de Chile for the hospitality extended him during his visit in January, 1986.

NOTE ADDED IN PROOF. In connection with our remark 4, it has been pointed out to us by Barry Simon that extension of the bound $\lambda_{2} / \lambda_{1} \leq 4$ to the line and half-line with $V(x) \rightarrow \infty$ as $|x| \rightarrow \infty$ follows from the given case by a limiting argument; in particular, the operator obtained by inserting infinite walls at $\pm a$ or 0 and $a$ (infinite walls $=$ Dirichlet boundary conditions) converges to the original operator in norm resolvent sense as $a \rightarrow \infty$ and this guarantees convergence of the eigenvalues. Since the bound holds for all finite-domain problems with Dirichlet boundary conditions it must continue to hold in the limit.

\section{REFERENCES}

1. R. Benguria, $A$ note on the gap between the first two eigenvalues for the Schrödinger operator, J. Phys. A 19 (1986), 477-478.

2. M. M. Crum, Associated Sturm-Liouville systems, Quart. J. Math. Oxford (2) 6 (1955), 121-127.

3. P. A. Deift, Applications of a commutation formula, Duke J. Math. 45 (1978), 267-310.

4. E. M. Harrell, unpublished, 1982.

5. V. A. Marchenko, The construction of the potential energy from the phases of the scattered waves, Dokl. Akad. Nauk SSSR 104 (1955), 695-698. MR 17, 740.

6. L. E. Payne, G. Pólya, and H. F. Weinberger, On the ratio of consecutive eigenvalues, J. Math. and Phys. 35 (1956), 289-298.

7. I. M. Singer, B. Wong, S.-T. Yau, and S. S.-T. Yau, An estimate of the gap of the first two eigenvalues in the Schrödinger operator, Ann. Scuola Norm. Sup. Pisa (4) 12 (1985), 319-333. 65211

Department of Mathematics, University of Missouri, Columbia, Missouri

Departamento de Física, Facultad de Ciencias Físicas y Matemáticas, UniVersidad DE Chile, CASilla $487 / 3$, SANTiago, Chile 